\section{Obstructive sleep apnea}

\author{
Matthew L. Ho, Steven D. Brass \\ UC Davis Department of Neurology, \\ Davis Medical Center, University \\ of California, California, USA
}

\section{Abstract}

Obstructive sleep apnea (OSA) affects millions of Americans and is estimated to be as prevalent as asthma and diabetes. Given the fact that obesity is a major risk factor for OSA, and given the current global rise in obesity, the prevalence of OSA will increase in the future. Individuals with sleep apnea are often unaware of their sleep disorder. It is usually first recognized as a problem by family members who witness the apneic episodes or is suspected by their primary care doctor because of the individual's risk factors and symptoms. The vast majority remain undiagnosed and untreated, despite the fact that this serious disorder can have significant consequences. Individuals with untreated OSA can stop breathing hundreds of times a night during their sleep. These apneic events can lead to fragmented sleep that is of poor quality, as the brain arouses briefly in order for the body to resume breathing. Untreated, sleep apnea can have dire health consequences and can increase the risk of hypertension, diabetes, heart disease, and heart failure. OSA management has also become important in a number of comorbid neurological conditions, including epilepsy, stroke, multiple sclerosis, and headache. Diagnosis typically involves use of screening questionnaires, physical exam, and an overnight polysomnography or a portable home study. Treatment options include changes in lifestyle, positive airway pressure, surgery, and dental appliances.

\section{Introduction}

The Greek word apnea means breathless or loss of breath. ${ }^{1}$ Sleep-disordered breathing (SDB) encompasses a heterogeneous group of sleep-related disorders that are characterized by abnormal pauses in breathing during sleep. There are two major types of SDB: obstructive sleep apnea (OSA) and central sleep apnea (CSA). Despite the difference in the actual cause of each type, in both cases, people with untreated sleep apnea stop breathing repeatedly during their sleep. Of the two types of sleep apneas characterized, OSA is the most common type, constituting greater than $85 \%$ of all cases of SBD; CSA is far less common. ${ }^{2}$
OSA is caused by a physical blockage of the airway; it results from airflow obstruction secondary to upper airway collapse or anatomic airway obstruction, even though the respiratory effort is still present. In the case of CSA, the airway is not blocked; the brain fails to signal the muscles to breathe and breathing is interrupted by a lack of respiratory effort. ${ }^{3}$

The clinical picture of OSA has long been recognized in medical literature. It was first described in 1918 by William Osler, a 20th century physician who coined the term Pickwickian syndrome in reference to a character in Charles Dickens's novel The Pickwick Papers. The character named Joe has all the classic symptoms of the condition. Joe is constantly hungry, red faced and always falling asleep in the middle of doing a task.

The object that presented itself to the eyes of the astonished clerk, was a boy - a wonderfully fat boy - habited as a serving lad, standing upright on the mat, with his eyes closed as if in sleep. 4

\section{Epidemiology}

OSA affects more than twelve million Americans. ${ }^{5}$ An epidemiological review by Young et al. estimates that 1 in 5 adults has at least mild OSA and 1 in 15 adults has at least moderate 0SA. ${ }^{6}$ Sleep apnea can affect anyone at any age, even children.7,8 OSA is becoming increasingly prevalent. However, because of the lack of awareness by the public and healthcare professionals, the vast majority remain undiagnosed and untreated. Data from the Wisconsin sleep cohort study of patients estimate that $93 \%$ of women and $82 \%$ of men with moderate-to-severe sleep apnea were undiagnosed. ${ }^{9}$ A follow-up publication from the Wisconsin Cohort Study five years later indicated that the prevalence of OSA in people aged 30-60 years was 9-24\% for men and 4-9\% for women. ${ }^{10,11}$ Primary risk factors for OSA include the male gender, those over age 40 , overweight persons or recent weight gain, and persons with a large neck size or small chin/jaw (Table 1).12

Epidemiological studies have consistently shown that body weight, and in particular BMI, is the strongest risk factor for OSA. It is estimated that about $70 \%$ of those with OSA are obese and that the prevalence of OSA in obese men and women is about $40 \% .13,14$ Twenty-six percent of patients with a BMI greater than 30 and $33 \%$ of those with a BMI greater than 40 have moderate 0SA. ${ }^{15}$ A large neck circumference is also associated with an increased risk of OSA. In fact, neck circumference of 15.7 in $(40 \mathrm{~cm})$ or greater may have a greater sensitivity and specificity than BMI in predicting OSA, regardless of the person's sex.16,17
Correspondence: Steven Brass, MD, MPH

Director of Neurology Sleep Medicine 4860 Y Street Suite 3700 Sacramento, CA 95817 Tel. +916.734 .6785 .

E-mail:steven.brass@ucdmc.ucdavis.edu

Key words: apnea, hypopnea, obstructive sleep apnea, apnea hypopnea index, respiratory disturbance index, respiratory event related arousals.

Received for publication: 23 August 2011. Accepted for publication: 30 August 2011.

This work is licensed under a Creative Commons Attribution NonCommercial 3.0 License (CC BYNC 3.0).

(C) Copyright M.L. Ho and S.D. Brass, 2011

Licensee PAGEPress, Italy

Neurology International 2011; 3:e15

doi:10.4081/ni.2011.e15

Age is also an important risk factor in the development of OSA; the prevalence is estimated to triple in individuals greater than 65 years of age compared with individuals aged 30-64 years. 18,19 Regarding gender, the male-tofemale ratio in community-based studies is about $3: 1 .{ }^{20}$ It is theorized that body fat distribution predispose men to OSA and that sex hormones play a role in the modulation of upper airway musculature.

As indicated above, the incidence of OSA among male and female are 3:1 prior to menopause. However, the incidence of OSA is equal amongst males and females following menopause. There is at least a threefold increase in risk of OSA among postmenopausal women compared to premenopausal women. ${ }^{21,22}$ In addition, there is a lower prevalence of OSA among postmenopausal women who were on hormone replacement therapy. ${ }^{23}$ Interestingly, there does not appear to be any difference in prevalence between males and females prior to puberty.

Structural factors related to craniofacial bony anatomy can predispose patients to pharyngeal collapse during sleep, including retrognathia and micrognathia, mandibular hypoplasia, and high-arched palate. ${ }^{24,25}$ Imaging studies have demonstrated that patients with OSA have compromised pharyngeal lumens that predispose them to collapse. ${ }^{26}$ This was independently confirmed under general anesthesia that simulates the total muscle paralysis seen during REM sleep. ${ }^{27}$ Patients with Down syndrome, Marfan syndrome, and Prader-Willi syndrome are particularly at risk due to these structural factors.

In terms of ethnicity, African American individuals appear to be more predisposed to OSA than Caucasians, with an odds ratio of 3 in chil- 
dren younger than 14 and 2 in adults greater than $25 .{ }^{28}$ Individuals of Asian descent, particularly Chinese individuals, have a more crowded upper airway and retrognathia compared with Caucasians. OSA has a prevalence rate similar in the Asian population sample to those in Caucasians despite a lower obesity rate. Asians have an increased risk of developing OSA, regardless of weight or neck circumference, as well as greater disease severity. ${ }^{29}$

\section{Pathophysiology}

The International Classification of Sleep Disorders, second edition (ICSD-2) was published by the American Academy of Sleep Medicine to standardize definitions and create a systematic approach to the diagnosis of sleep disorders. The ICSD-2 subdivides sleep disorders into eight major categories, one of which is sleep-disorder breathing (SDB). ${ }^{30}$

The ICSD-2 further classifies SDB into three basic categories: CSA syndromes, OSA syndromes, and sleep-related hypoventilation/ hypoxic syndromes. OSA involves a complete cessation or a significant decrease in airflow in the presence of breathing effort. CSA is the cessation of airflow with an absence of breathing effort. Breathing effort is measured by abdominal and/or chest movement.

The human airway is composed of soft tissue that can collapse during REM sleep, when the muscle tone of the body relaxes. Two major factors likely contribute to OSA pathophysiology: i) craniofacial structural abnormalities can predispose patients to OSA; this has been discussed previously and has been recognized as a primary risk factor for OSA; ii) larger soft tissue mass or abnormal tissue deposits can also increase extraluminal tissue pressure and lower the threshold for airway collapse (Figure 1). In normal, nonobese individuals without OSA, muscle relaxation during sleep does not completely collapse the airway (normal). However, airway collapse can occur during muscle relaxation when there is a pathological

Table 1. Primary risk factors for obstructive sleep apnea.

Primary risk factors for sleep apnea

Weight gain or being overweight with a BMI $>30$ $\mathrm{kg} / \mathrm{m}^{2}$

Neck circumference [ $\geq 17$ in $($ or $43.2 \mathrm{~cm})$ in men; $\geq 16$ in or $(40.6 \mathrm{~cm})$ in women]

Age $>40$

Male gender

Structural factors related to craniofacial anatomy Ethnicity

Family history of sleep apnea increase in tissue pressure, as a product of extra soft tissue mass (in a normal-sized enclosure) and/or structural limitations (small maxillary or mandibular compartment) with normal tissue mass (Figure 1). ${ }^{31}$ Chronically, this dysfunction can cause problems with the regulation of pharayngeal dilator muscle activation (which plays an important role in maintaining airway patency) in patients with OSA.

OSA is thus characterized by the partial or total collapse of the pharyngeal airway during sleep and the need to arouse to resume ventilation. ${ }^{32}$ In adults, the obstruction typically occurs at the level of the oropharynx (uvula/soft palate or tongue).33,34 Chronic severe OSA can result in prolonged hypoxemia, sleep deprivation, and other complications. With most apneic events, the brain briefly arouses in order for the body to resume breathing, but consequently, sleep is extremely fragmented and of poor quality.

Several additional anatomical factors play a role in OSA. These include the position that the patient sleeps in, airway reactivity and airway secretions. Position can have a strong influence on airway patency. Because the airway is collapsible, gravitational forces can cause the retropulsion of the tongue and soft palate while laying supine, thus generating increased positive tissue pressure and narrowing the airway. 35 For this reason, OSA worsens in the supine sleeping position for most individuals. ${ }^{36}$

\section{Evaluation}

Individuals with OSA are rarely aware of their sleep disorder, even upon arousal. ${ }^{37}$ Sleep apnea is usually recognized as a problem by family members who witness the apneic episodes or by a primary care doctor because of the individual's risk factors and symptoms. Most commonly, patients present with vague complaints. Clinical symptoms can include excessive daytime sleepiness (EDS) that usually begins during quiet activities (eg, reading, watching television), daytime fatigue, feeling tired despite a full night's sleep, morning headaches, personality and mood changes, dry or sore throat, gastroesophageal reflux, and sexual dysfunction.

Snoring is a common finding in individuals with OSA. Although not everyone who snores is experiencing sleep apnea, snoring in combination with obesity has been found to be highly predictive of OSA risk. ${ }^{38}$ The volume of the snoring is not indicative of the severity of obstruction. However, snoring with witnessed apneas has a $94 \%$ specificity for OSA.

The diagnosis of OSA is based on the evaluation of clinical symptoms and risk factors, as well as a formal sleep study evaluation (polysomnography, or a portable home based test). Individuals should be evaluated appropriately with screening questionnaires, as well as a physical examination.

The Epworth sleepiness scale (ESS) has been universally adopted as an effective screening method to monitor for clinical symptoms of sleep apnea. This questionnaire is used to help determine how frequently the patient is likely to doze off in 8 frequently encountered situations (e.g., as a passenger in a car, sitting quietly after lunch, etc). A 2003 study showed that an ESS score of 12 or greater is considered abnormal and would warrant a more formal evaluation. ${ }^{39}$ However, the

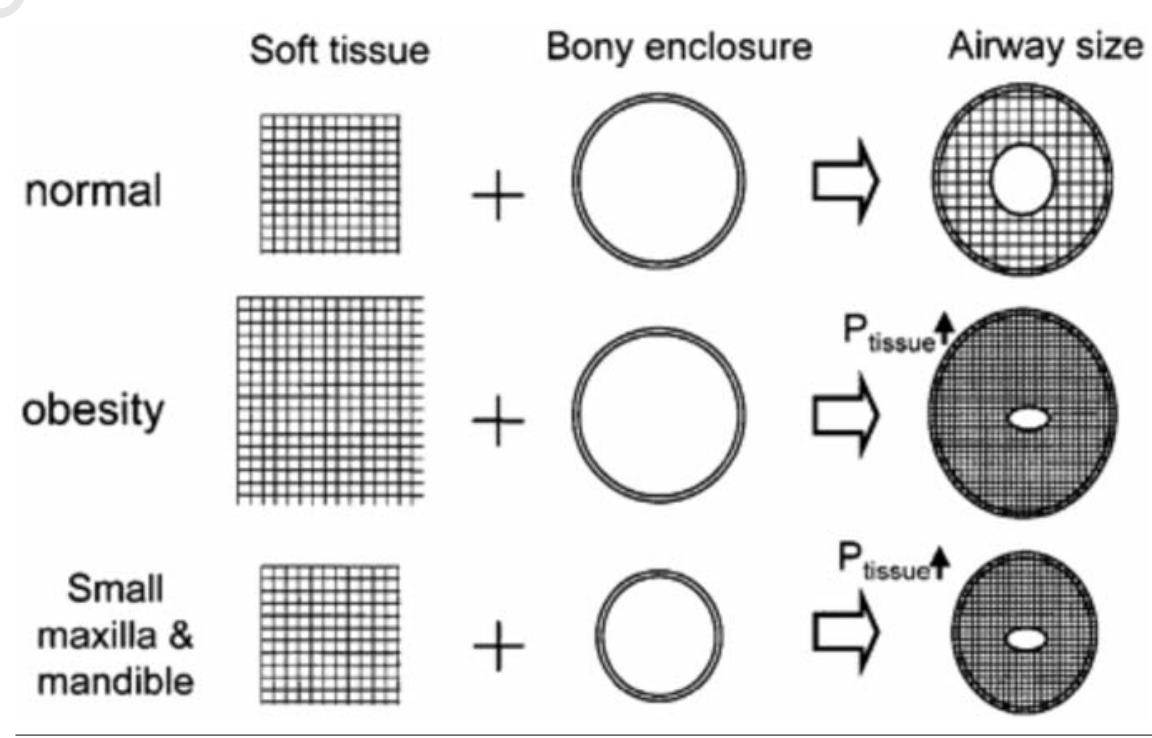

Figure 1. Reprinted with permission of the American Thoracic Society. Copyright (c) 2011 American Thoracic Society. White D. Pathogenesis of obstructive and central sleep apnea. Am J Resp Crit Care Med. 2005; 172: 1363-1370. Official Journal of the American Thoracic Society. 
ESS is still a subjective self assessment measure and may be inaccurate for a number of reasons. Therefore, if a patient has multiple risk factors for sleep apnea, the individual should be sent for further evaluation if there is a suspicion of sleep apnea despite a low ESS.

Another effective screening tool that has been used in the primary care population is the Berlin questionnaire. ${ }^{40}$ Survey questions address snoring behavior, EDS/fatigue, and history of obesity or hypertension. The sensitivity of the Berlin questionnaire with regards to high-risk patients having sleep apnea was $86 \%$. Another screening tool called the STOP $B A N G$ questionnaire was developed to screen for the most common risk factors seen specifically in OSA. The term refers to a mnemonic that represents 8 factors: Snoring, Tiredness, Observed apneas, elevated blood Pressure, BMI (greater than $35 \mathrm{~kg} / \mathrm{m}^{2}$ ), Age (greater than 50), Neck circumference (greater than 40 $\mathrm{cm}$ ), and Gender (male). Patients receive a point for each positive risk factor, and those whose scores are equal to or greater than 3 have a higher likelihood of having OSA. The sensitivities of the STOP BANG questionnaire for mild, moderate, and severe sleep apnea were $83.6 \%, 92.9 \%$, and $100 \%$, respectively.

The physical examination is frequently normal in patients with OSA, other than the presence of obesity, an enlarged neck circumference, and/or structural craniofacial bony abnormalities. There are other medical conditions that may be associated with the develop- ment of OSA, including hypothyroidism and acromegaly. Both are associated with macroglossia and increased soft tissue mass in the pharyngeal region, and patients should be routinely screened for these conditions as well.

Individuals should have a routine evaluation of their upper airway. The Mallampati score has been used for years to identify patients at risk for difficult tracheal intubation. It is now also used commonly by sleep physicians to evaluate for risk of OSA. The classification provides a score of 1 thru 4 based on the anatomic appearance of the airway seen when an individual opens his mouth (Figure 2). Studies have shown that for each 1 score increase in the Mallampati score, the number of apneic events increase. 42

The next step in the assessment is a formal sleep evaluation. An overnight polysomnography (PSG) is recorded during the normal sleeping hours of a patient. Patients sleep 6 to 8 hours before either awakening spontaneously or being awokened. The goal of the PSG is to quantify the amount of time spent in various stages of sleep during the night and to document clinically relevant events such as cardiopulmonary abnormalities and/or changes in sleep stages. The standard PSG includes limited multi-channel recording of an individual's electroencephalography (EEG) to assess for sleep architecture, sleep stages and arousals. In addition, surface electromyography of the chin and all four limbs (to assess for movement), electro-oculogram, airflow, pulse
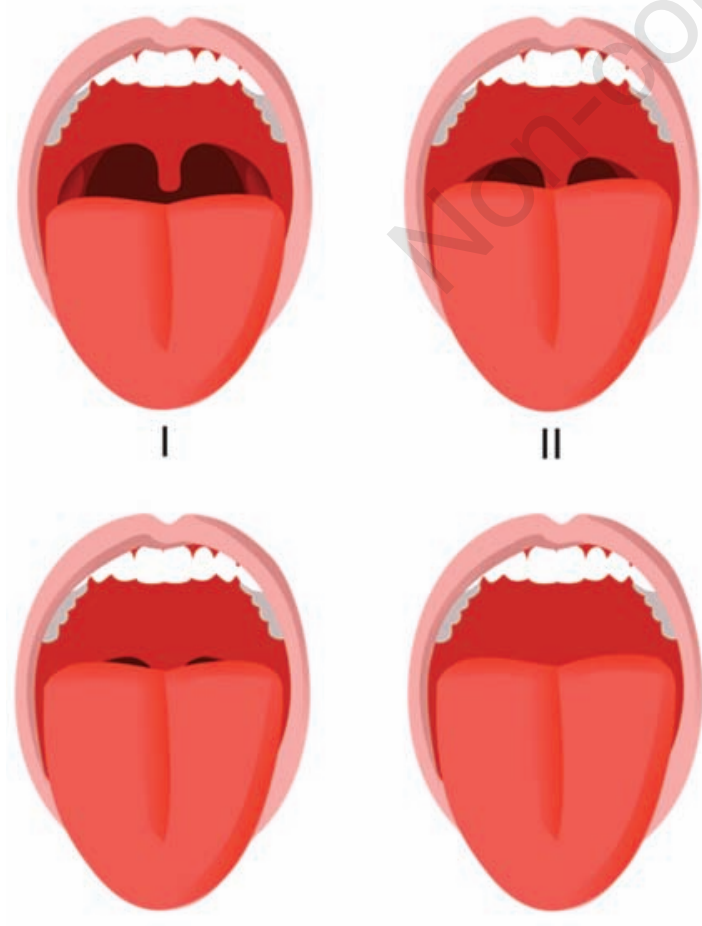

III
Figure 2. Mallampati score. Author Jmarchn. January 29, 2011. Permission is granted to copy, distribute and/or modify this document under the terms of the GNU Free Documentation License, Version 1.2 or any later version published by the Free Software Foundation. [online] Available from: http://en. wikipedia.org/wiki/Malla mpati_score.

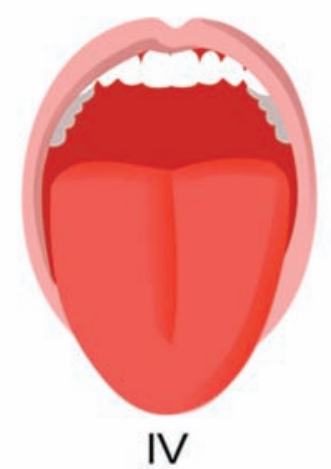

oximetry, respiratory effort, electrocardiographic (ECG) tracings, body position, and snoring are all accumulated.

The portable home-based sleep study has recently emerged as an alternative to the PSG. It assesses for oximetry, thoracic and abdominal movements, and body position. The advantage to this study is that it can be performed overnight in the comfort of the individual's home and is more cost effective in comparison to the formal PSG. In one study, normal overnight oximetry was very sensitive in excluding 0SA. ${ }^{43,44}$ However, the main limitation of the portable home sleep study is that it does not have the EEG component; it is therefore not able to assess an individual's sleep architecture and will not be able to assess arousals based on changes in sleep stages and can therefore underestimate the prevalence of OSA. It is thus indicated for use as an alternative to PSG for the diagnosis of OSA in patients with a high pretest probability of moderate to severe OSA and no other comorbid sleep disorders or major comorbid medical disorders. 45 Patients should be excluded if they have congestive heart failure, chronic obstructive pulmonary disease, neuromuscular disease, use certain medications (potent narcotics), or may have another suspected causes for sleepiness.

\section{Diagnosis}

The American Academy of Sleep Medicine (AASM) defines an apnea as a cessation in airflow lasting at least $10 \mathrm{sec}$; apneic episodes can last anywhere from $10 \mathrm{sec}$ to min, and may occur multiple times per hour.46 Hypopnea is defined as a recognizable transient reduction (but not complete cessation) of breathing for at least10 sec. This differs from apnea in that there remains some flow of air. In the context of sleep disorders, a hypopnea event is only considered to be clinically significant if there is a $30 \%$ or more reduction in flow with an associated $4 \%$ or greater desaturation in 02 level, lasting for 10 seconds or longer, or if it is associated with an arousal or fragmentation of sleep. Apneas and hypopneas are both considered in assessing the severity of a person's sleep disorder. 47

The Apnea-Hypopnea Index (AHI) is an index used to assess the severity of sleep apnea based on the total number of apneas and hypopneas occurring per hour of sleep. In general, an individual is considered to have an OSA syndrome if they demonstrate an AHI of at least 5 with the presence of daytime symptoms or AHI of 15 or more independent of symptoms. The AHI can also be used to stratify the severity of the disease; an AHI of 5-15 is classified as mild, 15-30 is considered moderate, and greater than 30 is considered severe. 48 
Another measure that is often used is the Respiratory Disturbance Index (RDI). Like the AHI, RDI measures respiratory events; however, it also includes respiratory event related arousals (RERAs). RERAs are arousals from sleep that do not technically meet the definitions of apneas or hypopneas, but do disrupt sleep. Some research studies have found that $30 \%$ of symptomatic patients would have been left untreated if the AHI were used rather the RDI. ${ }^{49}$ Please see Figure 5 for a simplified guideline for the evaluation, diagnosis and appropriate treatment options for OSA management.

\section{Complications and comorbidities}

Untreated, OSA can have a profound effect on the body and can contribute to multiple health problems (Table 2). It is associated with cardiovascular disease, ${ }^{50,51}$ hypertension, ${ }^{52-54}$ stroke, 55 arrhythmias, and insulin resistance. Patients with OSA have a 30\% higher risk of heart attack or death than those unaffected. ${ }^{58}$ Hypertension that is primarily caused by OSA (in contrast to essential hypertension) is distinctive in that the blood pressure does not drop significantly when the individual is sleeping. ${ }^{59}$ OSA that remains untreated can also have a negative effect on memory. Research has recently shown that individuals with OSA have mammillary bodies that were nearly 20 percent smaller on MRI. ${ }^{60}$ This decreased tissue mass may be related to the memory problems seen in chronic OSA.

OSA can have broader cognitive impairments, including sustained attention, working memory, visuospatial learning, motor performance, and executive functioning. ${ }^{61,62}$ In particular, patients with OSA have been shown to have impaired judgment, prolonged reaction time and vision problems, all of which can compromise work and driving ability. ${ }^{6}$ OSA increases motor vehicle accident risk by 2 - to 3 -fold, independent of EDS or AHI score. 64 Behavior and mood can also be profoundly affected by a lack of the adequate restful sleep seen in sleep apnea, including irritability, aggressiveness, lack of attentiveness, as well as depression. 65

There has been increasing recognition of OSA as a comorbid condition in a number of neurological disorders, including epilepsy, stroke, multiple sclerosis, and headache. OSA appears to be more prevalent in patients with epilepsy than in the general population and untreated OSA may theoretically worsen seizure control by increasing seizure burden through sleep disruption/deprivation. 66 OSA also increases the risk of stroke and appears to compromise rehabilitation following strokes. ${ }^{67}$ It is theorized that OSA may directly cause direct neural injury or cause extension of vascular penumbra via hypoxia and ischemia in stroke. 68

There appears to be a higher incidence of OSA in the multiple sclerosis (MS) population, but this has been poorly studied in small prospective series. It is unclear at this time if this comorbidity association is due to MS lesions, symptomatic medications used for pain and spasticity, etc. ${ }^{69}$ Chronic and morning headaches are a relatively common symptom in OSA, and OSA may aggravate the frequency and severity of migraines. ${ }^{70}$ OSA treatment has been shown to reduce cluster headache frequency in patients with OSA and comorbid cluster headaches. ${ }^{71}$ The conclusion regarding OSA as a comorbid condition in some neurological disorders is that neurologists should be mindful of the fact that OSA is more prevalent in their patient population and that practitioners should routinely screen for OSA in their patients.

\section{Treatment options}

The most common first step in the treatment of mild OSA is behavioral or lifestyle modification, including losing weight, avoiding alcohol, sleep aids or muscle relaxants, and quitting smoking. 72 Weight loss in overweight patients has been shown to decrease apneic episodes, ${ }^{2}, 73$ both directly and by reducing neck fat and nasopharyngeal crowding. ${ }^{75,76}$

If an individual's apneic episodes are mild and occur mainly during supine sleep, then patients are advised to take measures to avoid sleeping supine. 77,78 The most common position that apneic and hypopnic episodes are observed during sleep is in the supine position. These patients may also benefit from sleeping at a 30 -degree incline. ${ }^{79}$ Both these steps may help prevent the tongue and palate from falling backwards and prevents the gravitational collapse of the airway.

Along with lifestyle modifications, there are other treatment options of OSA.

\section{Positive airway pressure}

For moderate to severe sleep apnea, the use of a continuous positive airway pressure (CPAP) is the first line therapy. ${ }^{80}$ CPAP uses continuous pressurized air flow to keep the individual's airway open during sleep. The amount of pressure used is initially titrated during the PSG (split test) based on the patient's comfort and lowest pressure required to decrease apneic and hypopneic episodes. CPAP therapy is the most effective treatment option in reducing apneas in 0SA.81 It has been shown to improve AHI, RDI, sleep architecture, EDS, neurobehavioral performance and cardiovascular morbidity (hypertension).
Table 2. Long term health problems associated with untreated obstructive sleep apnea.

Health problems associated with sleep apnea

Hypertension

Stroke

Diabetes

Heart failure

Cardiac arrhythmia

Myocardial infarction

Obesity

Depression

Worsening of Attention Defficit Hyperactivity Disorder (ADHD)

However, noncompliance is common and may be as high as $40 \%$, due mainly to discomfort. ${ }^{82,83}$

Newer models of CPAP devices humidify the air, which decreases dry mouth and makes usage more comfortable. 84 In addition, newer models have automatic positive airway pressure options. This modality automatically titrates the amount of pressure delivered to the patient to the minimum required to maintain an unobstructed airway on a breath-by-breath basis by measuring the resistance in the patient's breathing, thereby giving the patient the precise pressure required at a given time. 85

BiPAP (variable/bilevel positive airway pressure) provides two levels of pressure: a higher inspiratory positive airway pressure (IPAP) and a lower expiratory positive airway pressure (EPAP) for easier exhalation. This modality appears to be better tolerated and individuals may find it easier to use; it is used in cases when patients cannot tolerate CPAP, for patients with chronic $\mathrm{CO}_{2}$ retention as well as sleep apnea, and for patients with neuromuscular disease who need some assistance with nocturnal ventilation. BiPAP may also be more useful in CSA. ${ }^{86}$

\section{Dental appliances}

Dentists specializing in sleep dentistry can make a custom-made mouthpiece that shifts the lower jaw forward, thereby maintaining an open airway (in theory). This approach can be successful in individuals with mild to moderate OSA but has been proven less effective for severe cases. ${ }^{87}$ In one study comparing CPAP to dental appliance in mild to moderate OSA, dental appliances decreased AHI from 21 (baseline) to 14, compared to a decreased AHI of 5 in patients using CPAP. 88

\section{Surgery}

Surgical treatment for OSA needs to be individualized in order to address all anatomical areas of obstruction. The most frequently uti- 
lized surgery treatment is the uvulopalatopharyngoplasty (UPPP or UP3). These surgeries aim to address pharyngeal obstruction by removing tissue in the back of the throat, including part of the uvula, the soft palate, the tonsils, the adenoids and pharynx (Figure 3). In a retrospective review comparing CPAP to surgery, there appeared to be superior results with CPAP compared to UPPP. ${ }^{89}$ Further studies have not been conclusive that this surgery has been effective at treating severe OSA. 90

Maxillomandibular advancement (MMA) is another type of surgery that has been used to treat OSA. This procedure aims to advance the maxilla and mandible, thereby pulling forward the anterior pharyngeal tissues attached to the maxilla, mandible and hyoid to structurally enlarge the retrolingual and retropalatal spaces (Figure 4). ${ }^{91}$ It is considered the most effective surgery for OSA patients, because it increases the posterior airway space. ${ }^{92}$ In a study in 2008, it was noted that MMA surgery led to a significant increase in general productivity, social outcome, activity level and sex. ${ }^{93}$

In addition, there are specialized techniques that are available for correction of specific regions or obstruction, including the nasal passages (septoplasty and turbinate surgery), the soft palate (implantation of Dacron pillars) and the tongue. However, there have not been clinical trials or current evidence to support the use of these techniques in the treatment of OSA.

\section{Pharmacological management}

Medications are generally not a part of the primary treatment of OSA. Modafinil is approved by the FDA for use in patients with OSA who have residual daytime sleepiness.
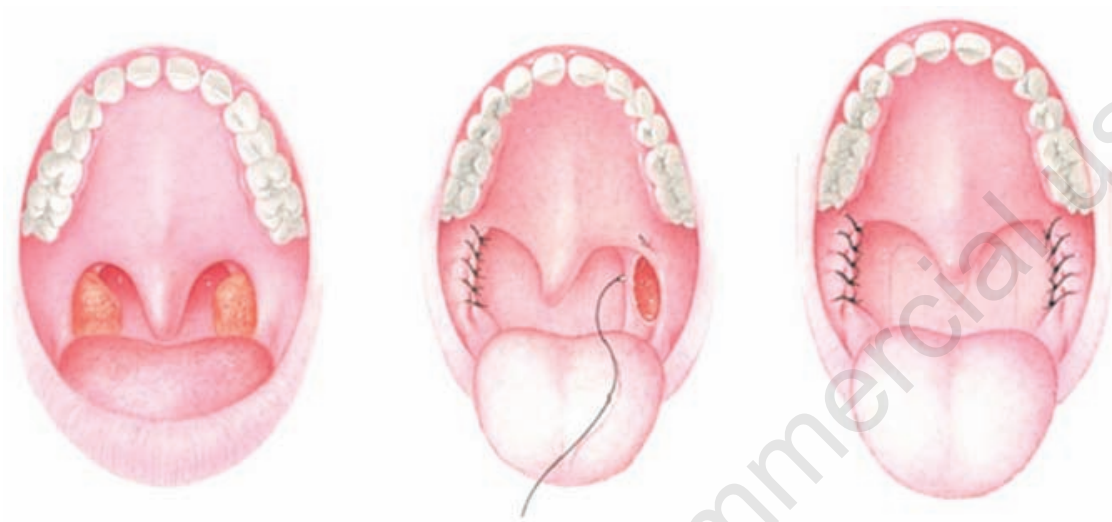

Figure 3. Reprinted with permission of the American Thoracic Society. Copyright (c) 2011 American Thoracic Society. Won C, Li K, Guilleminault C. Surgical treatment of obstructive sleep apnea: upper airway and maxillomandibular surgery. Proc Am Thorac Soc. 2008; 5: 193-199. Official Journal of the American Thoracic Society.

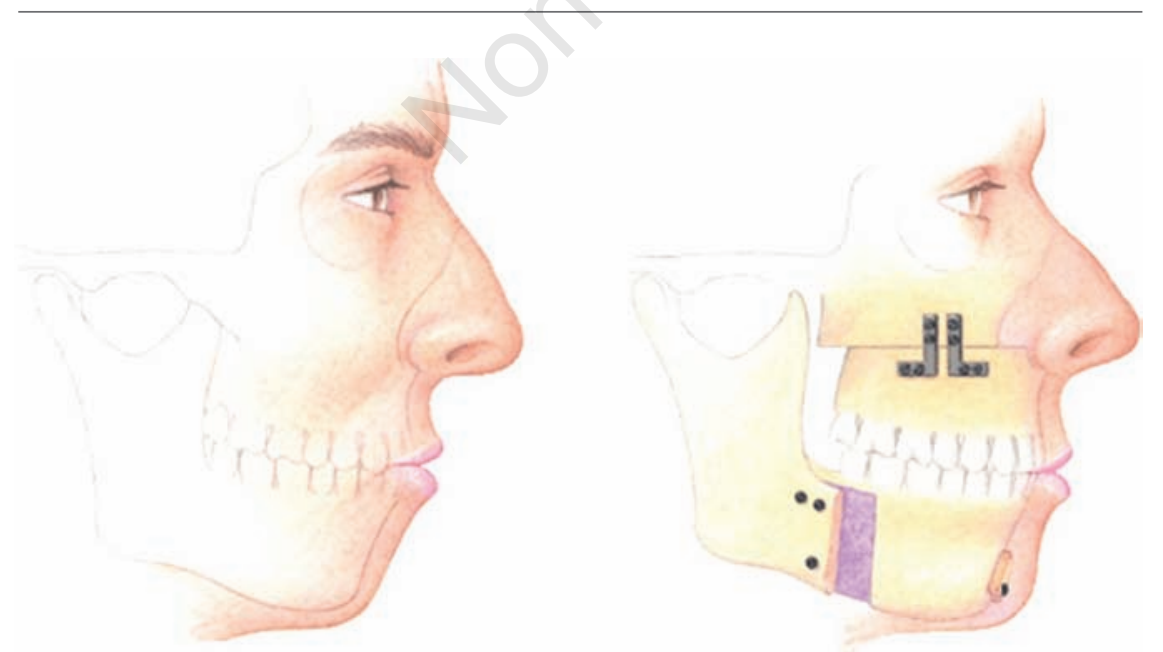

Figure 4. Reprinted with permission of the American Thoracic Society. Copyright (c) 2011 American Thoracic Society. Won C, Li K, Guilleminault C. Surgical treatment of obstructive sleep apnea: upper airway and maxillomandibular surgery. Proc Am Thorac Soc. 2008; 5: 193-199. Official Journal of the American Thoracic Society.

Armodafinil, the R-enantiomer of modafinil, is now FDA approved for use as well. The American Academy of Sleep Medicine (AASM), in a clinical review of medical therapies for OSA, recommended Modafinil as a standard treatment of residual excessive daytime sleepiness in patients with OSA despite maximal management of CPAP. Protriptyline was not recommended as a primary treatment for OSA, although it was acknowledged that it may induce moderate improvement in the $\mathrm{AHI}$ in patients with OSA and may be used as a second line treatment option. Aminophylline, theophylline, SSRIs and estrogen were not recommended for treatment of patients with OSA given that there was no consistent evidence of their effectiveness. ${ }^{94}$

\section{Conclusions}

In the context of the current epidemic of obesity, the prevalence and consequences of OSA will likely increase in the coming years. Given the aging population, the number of neurological patients with OSA and comorbid stroke, epilepsy, headache, and cognitive decline will also likely rise. OSA can significantly affect physical health, mental health and emotional well-being. Fortunately, screening methods for sleep apnea have improved, and there are now very effective means of diagnosis and treatment. Treatment can lead to a beneficial impact on a patient's health and quality of life. Several treatment options exist, and research into additional options continues.

The medical community faces many hurdles regarding the development of adequate early screening and appropriate treatment of OSA. An important next steps in understanding and treating OSA is to be proactive and develop better screening methods and properly treat patients at risk (Figure 5).

It is imperative that the medical community understand that the effects of OSA is farreaching, and early detection/treatment will be beneficial to individual patients, as well as a cost-effective public health measure to reduce morbidity and mortality. Physicians of all specialties should screen for the presence of sleep disturbances and consider referral to a sleep specialist when indicated. Neurologists, in particular, should be mindful of comorbid OSA in their patient population, since prompt identification and treatment of OSA may reduce health risk and improve neurological functioning.

\section{References}

1. Oxford English Dictionary. Oxford 


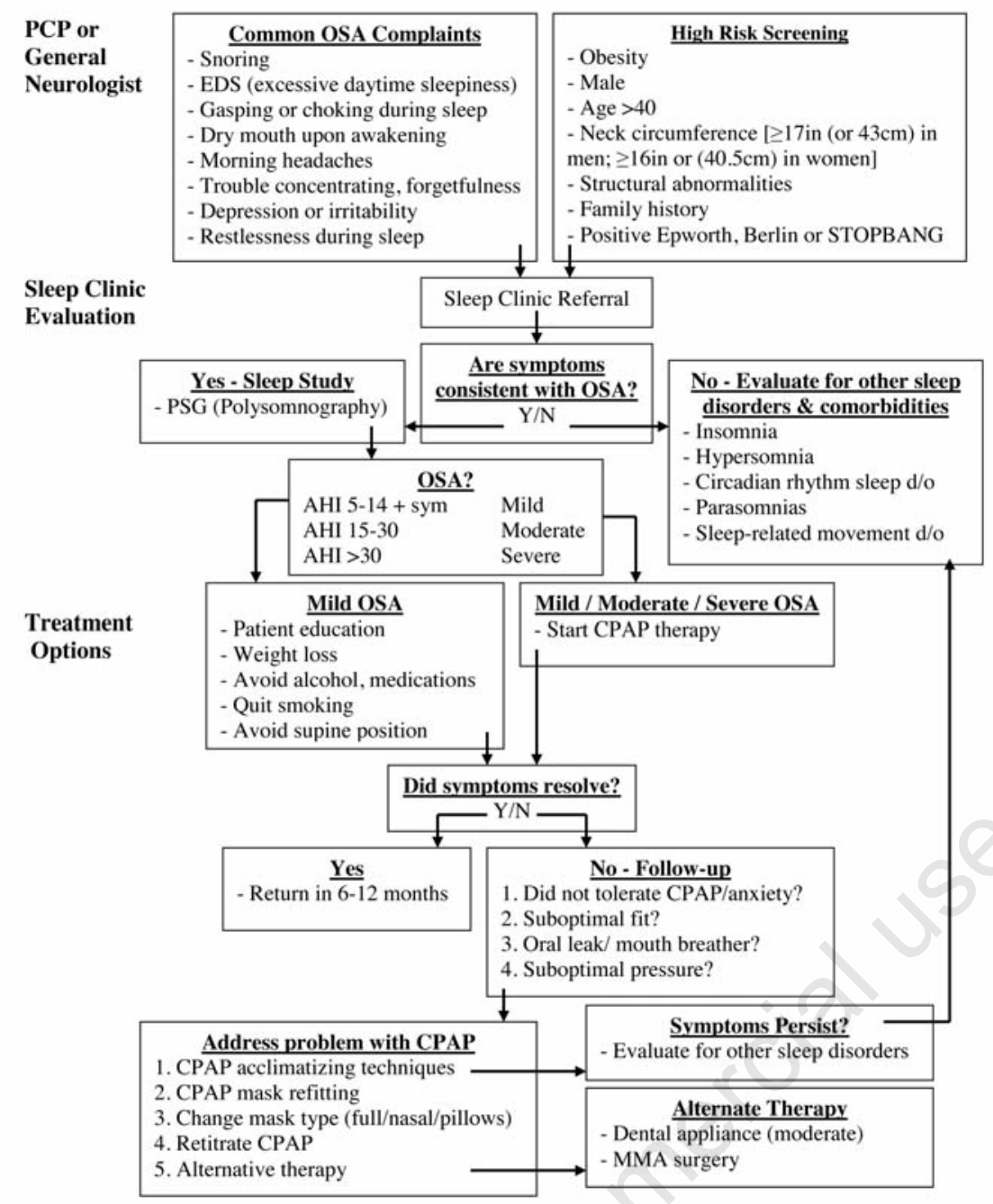

Figure 5. Evaluation of OSA. Approach to initiation, management, and follow-up of treatment.

University Press. 2011. http://www.oed. com/viewdictionaryentry/Entry/9226.

2. Morgenthaler T, Kagramanov V, Hanak V, et al. Complex sleep apnea syndrome: is it a unique clinical syndrome? Sleep 2006; 29:1203-9.

3. White D. Pathogenesis of obstructive and central sleep apnea. Am J Resp Crit Care Med 2005;172:1363-70.

4. Dickens C, The Pickwick Papers (The Posthumous Papers of the Pickwick Club). Penguin Classics, London, 2000.

5. "Sleep Apnea: Who Is At Risk for Sleep Apnea?" NHLBI: Health Information for the Public. US Department of Health and Human Services. Avaible at: http://www.nhlbi.nih.gov/health/dci/Diseas es/SleepApnea/SleepApnea_WhoIsAtRisk. html.

6. Young T, Peppard P, Gottlieb D. Epidemiology of obstructive sleep apnea: a population health perspective. Am J Resp
Crit Care Med. 2002; 65:1217-39.

7. Kalra M, Chakraborty R. Genetic susceptibility to obstructive sleep apnea in the obese child. Sleep Medicine 2007:8:169-75.

8. Bixler E, Vgontzas A, Lin H, et al. Blood pressure associated with sleep-disordered breathing in a population sample of children. Hypertension 2008;52:841-6.

9. Young T, Evans L, Finn L. Estimation of the clinically diagnosed proportion of sleep apnea syndrome in middle-aged men and women. Sleep 1997;20:705-6.

10. Young T, Peppard P, Gottlieb D. Epidemiology of obstructive sleep apnea: a population health perspective. Am J Resp Crit Care Med. 2002;165:1217-39.

11. Kapur V, Blough D, Sandblom R, et al. The medical cost of undiagnosed sleep apnea. Sleep 1999;22:749-55.

12. Tishler P, Larkin E, Schluchter M, et al. Incidence of sleep disordered breathing in an urban adult population: the relative importance of risk factors in the development of sleep-disordered breathing. JAMA 2003;289: 2230-37.

13. Malhotra A, White D. Obstructive sleep apnea. Lancet 2002;360:237-45.

14. Young T, Peppard P, Gottlieb D. Epidemiology of obstructive sleep apnea: a population health perspective. Am J Resp Crit Care Med 2002;165:1217-39.

15. Valencia-Flores M, Orea A, Castano V, et al. Prevalence of sleep apnea and electrocardiographic disturbances in morbidly obese patients. Obes Res 2000;8:262-9.

16. Tsai W, Remmers J, Brant R, et al. A decision rule for diagnostic testing in obstructive sleep apnea. Am J Resp Crit Care Med 2003;167:1427-32.

17. Davies R, Ali N, Stradling J. Neck circumference and other clinical features in the diagnosis of the obstructive sleep apnoea syndrome. Thorax 1992;47:101-5.

18. Young T, Palta M, Dempsey J, et al. The occurrence of sleep-disordered breathing among middle-aged adults. N Engl J Med Apr 1993;328:1230-5.

19. Young T, Shahar E, Nieto F, et al. Predictors of sleep-disordered breathing in community-dwelling adults: the Sleep Heart Health Study. Arch Intern Med 2002;162:893-900.

20. Redline S, Kump K, Tishler P, et al. Gender differences in sleep disordered breathing in a community-based sample. Am J Resp Crit Care Med 1994;149:722-6.

21. Young T, Finn L, Austin D, et al. Menopausal status and sleep-disordered breathing in the Wisconsin Sleep Cohort Study. Am J Resp Crit Care Med 2003;167:1181-85.

22. Bixler E, Vgontzas A, Lin $\mathrm{H}$, et al. Prevalence of sleep-disordered breathing in women: effects of gender. Am J Resp Crit Care Med 2001;163:608-13.

23. Young T, Skatrud J, Peppard P. Risk factors for obstructive sleep apnea in adults. JAMA 2004;291:2013-6.

24. Fogel R, Malhotra A, White D. Sleep. 2: pathophysiology of obstructive sleep apnoea/hypopnoea syndrome. Thorax 2004;59:159-63.

25. Watanabe $\mathrm{T}$, Isono $\mathrm{S}$, Tanaka $\mathrm{A}$, et al. Contribution of body habitus and craniofacial characteristics to segmental closing pressures of the passive pharynx in patients with sleep disordered breathing. Am J Resp Crit Care Med 2002;165: 260-5.

26. Schwab R, Pasirstein M, Pierson R, et al. Identification of upper airway anatomic risk factors for obstructive sleep apnea with volumetric magnetic resonance imaging. Am J Resp Crit Care Med 2003;168:522-30.

27. Isono S, Remmers J, Tanaka A, et al. Anatomy of pharynx in patients with 
obstructive sleep apnea and in normal subjects. J App Physiol 1997;82:1319-26.

28. Cakirer B, Hans M, Graham G, et al. The relationship between craniofacial morphology and obstructive sleep apnea in whites and in African-Americans. Am J Resp Crit Care Med 2001;163:947-50.

29. Ip M, Lam B, Lauder I, et al. A community study of sleep-disordered breathing in middle-aged Chinese men in Hong Kong. Chest 2001;119:62-9.

30. American Academy of Sleep Medicine. The international classification of sleep disorders. Sateia M, editor. Diagnostic and coding manual. 2nd edition. Westchester (IL): American Academy of Sleep Medicine; 2005. pp. 1-297.

31. White D. Pathogenesis of obstructive and central sleep apnea Am J Resp Crit Care Med 2005;172:1363-70.

32. Kuna S, Sant'Ambrogio G. Pathophysiology of upper airway closure during sleep. JAMA 1991; 266:1384-9.

33. Patil S, Schneider H, Schwartz A, et al. Adult obstructive sleep apnea: pathophysiology and diagnosis. Chest 2007;132:32537.

34. Eckert D, Malhotra A, Pathophysiology of adult obstructive sleep apnea, Proc Am Thorac Soc 2008; 144-53.

35. Fouke J, Strohl K. Effect of position and lung volume on upper airway geometry. J Appl Physiol 1987;63:375-80.

36. Xiheng G, Chen W, Hongyu Z, et al. The Study of The Influence of Sleep Position on Sleep Apnea. Cardinal Health. 2003.

37. Sleep Apnea: Key Points. NHLBI: Health Information for the Public. U.S. Department of Health and Human Services.

38. Morris L, Kleinberger A, Lee K, et al. Rapid risk stratification for obstructive sleep apnea, based on snoring severity and body mass index. Otolaryngology-Head and Neck Surgery 2008;139:615-8.

39. Benbadis S, Mascha E, Perry M, et al. Association between the Epworth sleepiness scale and the multiple sleep latency test in a clinical population. Ann Intern Med 1999;130:289-92.

40. Netzer N, Stoohs R, Netzer C, et al. Using the Berlin Questionnaire to identify patients at risk for the sleep apnea syndrome. Ann Intern Med 1999;131:485-91.

41. Chung F, Yegneswaran B, Liao P, et al. STOP questionnaire: A tool to screen patients for obstructive sleep apnea. Anesthesiology 2008;108: 812-21.

42. Nuckton T, Glidden D, Browner W, et al. Physical examination: Mallampati score as an independent predictor of obstructive sleep apnea. Sleep 2006; 29:903-8.

43. Sériès F, Marc I, Cormier Y, et al. Utility of nocturnal home oximetry for case finding in patients with suspected sleep apnea hypopnea syndrome. Ann Intern Med 1993;119:449-53.

44. McNicholas W, Lévy P. Portable monitoring in sleep apnoea: the way forward? Eur Resp J 2011;37:749-51.

45. Collop N, Anderson W, Boehlecke B, et al. Clinical guidelines for the use of unattended portable monitors in the diagnosis of obstructive sleep apnea in adult patients. Portable Monitoring Task Force of the American Academy of Sleep Medicine. J Clin Sleep Med 2007;3:737-47.

46. Sleep Apnea: What Is Sleep Apnea?. NHLBI: Health Information for the Public. U.S. Department of Health and Human Services. 2009-05.

47. American Academy of Sleep Medicine. International Classification of Sleep Disorders. In: Diagnostic and Coding Manual. Second Edition. Westchester, Ill: American Academy of Sleep Medicine; 2005.

48. Ruehland W, Rochford P, O'Donoghue F, et al. The new AASM criteria for scoring hypopneas: impact on the apnea hypopnea index. Sleep. February 2009;32:150-7.

49. Howard M, Desai A, Grunstein R, et al. Sleepiness, sleep-disordered breathing, and accident risk factors in commercial vehicle drivers. Am J Resp Crit Care Med 2004;170:1014-21.

50. Somers V, White D, Amin R, et al. Sleep apnea and cardiovascular disease: an American Heart Association/American College of Cardiology Foundation Scientific Statement from the American Heart Association Council for High Blood Pressure Research Professional Education Committee, Council on Clinical Cardiology, Stroke Council, and Council on Cardiovascular Nursing. J Am Coll Cardiol 2008;52:686-717.

51. Parati G, Lombardi C, Narkiewicz K. Sleep apnea: epidemiology, pathophysiology, and relation to cardiovascular risk. Am J Phys Reg Integ Comp Physiol 2007;293: R1671-83.

52. Pepperell J, Ramdassingh-Dow S, Crosthwaite N. Ambulatory blood pressure after therapeutic and subtherapeutic nasal continuous positive airway pressure for obstructive sleep apnea: a randomized parallel trial. Lancet 2002; 359:204-10.

53. Faccenda J, Mackay T, Boon N, et al. Randomized, placebo-controlled trial of continuous positive airway pressure on blood pressure in the sleep apnea/hypopnea syndrome. Am J Resp Crit Care Med 2001;163:344-8.

54. Silverberg D, Iaina A, Oksenberg A. Treating obstructive sleep apnea improves essential hypertension and life. Am Fam Physician 2002;65:229-36.
55. Yaggi H, Concato J, Kernan W, et al. Obstructive sleep apnea as a risk factor for stroke and death. N Eng J Med 2005;353: 2034-41.

56. Leung R. Sleep-disordered breathing: autonomic mechanisms and arrhythmias. Progress in Cardiovascular Diseases 2009;51:324-38.

57. Aronsohn R, Whitmore H, Van Cauter E, et al. Impact of untreated obstructive sleep apnea on glucose control in type 2 diabetes. Am J Resp Crit Care Med 2010;181:507-13.

58. Shah N, Botros N, Yaggi H, et al. Sleep Apnea Increases Risk of Heart Attack or Death by $30 \%$. American Thoracic Society. May 20, 2007.

59. Grigg-Damberger M. Why a polysomnogram should become part of the diagnostic evaluation of stroke and transient ischemic attack. J Clin Neurophys 2006;23: 21-38.

60. Kumar R, Birrer BV, Macey P, et al. Reduced mammillary body volume in patients with obstructive sleep apnea. Neuroscience Letters 2008;438:330-4.

61. Ferini-Strambi L, Baietto C, Di Gioia M, et al. Cognitive dysfunction in patients with obstructive sleep apnea (OSA): Partial reversibility after continuous positive airway pressure (CPAP). Brain Res Bull 2003; 61:87-92.

62. Beebe D, Groesz L, Wells C, et.al. The neuropsychological effects of obstructive sleep apnea: a meta-analysis of norm-referenced and case-controlled data. Sleep 2003; 26:298-307.

63. Sassani A, Findley L, Kryger M, et al. Reducing motor-vehicle collisions, costs, and fatalities by treating obstructive sleep apnea syndrome. Sleep 2004;27:453-8.

64. Ellen R, Marshall S, Palayew M, et al. Systematic review of motor vehicle crash risk in persons with sleep apnea. J Clin Sleep Med 2006;2:193.

65. Schröder C, O'Hara R. Depression and Obstructive Sleep Apnea (OSA). Ann Gen Psychiatry 2004;4:13.

66. Malow B, Levy K, Maturen $\mathrm{K}$, et al. Obstructive sleep apnea is common in medically refractory epilepsy patients. Neurology 2000;55:1002-7.

67. Dyken M, Im K. Obstructive sleep apnea and stroke. Chest 2009;136:1668-77.

68. Louis E. Diagnosing and treating co-morbid sleep apnea in neurological disorders. Practical neurology 2010;9:26-30.

69. Brass S, Duquette P, Proulx-Therrien J, et.al. Sleep medicine reviews 2010;14:1219.

70. Rains J, Poceta J. Headache and sleep disorders: review and clinical implication for headache management. Headache 2006;46:1344-63. 
71. Nath Zallek S, Cheervin R. Improvement in cluster headache after treatment for obstructive sleep apnea. Sleep Med 2000;1:135-8.

72. How Is Sleep Apnea Treated?". National Heart, Lung, and Blood Institute. Avalaible at:http://www.nhlbi.nih.gov/health/dci/Dise ases/SleepApnea/SleepApnea_Treatments. html.

73. Kansanen M, Vanninen E, Tuunainen A, et al. The effect of a very low calorie diet induced weight loss on the severity of obstructive sleep apnoea and autonomic nervous function in obese patients with obstructive sleep apnoea syndrome. Clin Physiol 1998;18:377-85.

74. Rubinstein I, Colapinto N, Rotstein L, et al. Improvement in upper airway function after weight loss in patients with obstructive sleep apnea. Am Rev Resp Dis 1988;138:1192-5.

75. Suratt $\mathrm{P}$, McTier R, Findley L, et al. Changes in breathing and the pharynx after weight loss in obstructive sleep apnea. Chest 1987;92:631-7.

76. Hernandez T, Ballard R, Weil K, et al. Effects of maintained weight loss on sleep dynamics and neck morphology in severely obese adults. Obesity 2009;17:84-91.

77. Loord H, Hultcrantz E. Positioner - a method for preventing sleep apnea". Acta Oto-laryngologica. 2007;127:861-8.

78. Szollosi I, Roebuck T, Thompson B, et al. Lateral sleeping position reduces severity of central sleep apnea / Cheyne-Stokes respiration. Sleep 2006;29:1045-51.

79. Neill A, Angus S, Sajkov D, et al. Effects of sleep posture on upper airway stability in patients with obstructive sleep apnea. Am J Resp Crit Care Med 1997;155:199-204.
80. Epstein E,(Chair), Kristo D, Strollo Jr P. Clinical Guidelines for the Evaluation, Management and Long-term Care of Obstructive Sleep Apnea in Adults. J Clin Sleep Med 2009;5:263-79.

81. Gay P, Weaver T, Loube D, et al. Evaluation of positive airway pressure treatment for sleep related breathing disorders in adults. Sleep 2006;29:381-401.

82. Hsu AA, Lo C. Continuous positive airway pressure therapy in sleep apnea. Respirology 2003;8:447-54.

83. Stepnowsky Jr C, Moore P. Nasal CPAP treatment for obstructive sleep apnea: developing a new perspective on dosing strategies and compliance. J Psychosomatic Research 2003;54:599-605.

84. Ballard R, Gay P, Strollo P. Interventions to improve compliance in sleep apnea patients previously non-compliant with continuous positive airway pressure. J Clin Sleep Med 2007;3:706-12.

85. Vennelle M, White S, Riha $R$, et al. Randomized controlled trial of variablepressure versus fixed-pressure continuous positive airway pressure (CPAP) treatment for patients with obstructive sleep apnea/hypopnea syndrome (OSAHS). Sleep 2010;33:267-71.

86. Reeves-Hoche M, Hudgel D, Meck R, et al. Continuous versus bilevel positive airway pressure for obstructive sleep apnea. Am J Resp Crit Care Med 1995;151:443-9.

87. Machado M, Juliano L, Taga M, et al. Titratable mandibular repositioner appliances for obstructive sleep apnea syndrome: are they an option?. Sleep \& Breathing 2007;11:225-31.

88. Barnes M, McEvoy R, Banks S, et al. Efficacy of positive airway pressure and oral appliance in mild to moderate obstructive sleep apnea. Am J Resp Crit Care Med 2004;170:656-64.

89. Anand V, Ferguson P, Schoen L. Obstructive sleep apnea: comparison of continuous positive airway pressure and surgical treatment. Otolaryngology Head Neck Surgery 1991;105:382-90.

90. Schecthtman K, Sher A, Piccirillo J. Methodological and statistical problems in sleep apnea research: the literature on Uvulopalatopharyngoplasty. Sleep 1995;18:659-66.

91. Won C, Li K, Guilleminault C. Surgical treatment of obstructive sleep apnea: upper airway and maxillomandibular surgery. Proc Am Thorac Soc 2008;5:193-9.

92. Prinsell J. Maxillomandibular advancement surgery for obstructive sleep apnea syndrome. J Am Dental Assn 2002;133:1489-97.

93. Lye K, Waite P, Meara D, et al. Quality of life evaluation of maxillomandibular advancement surgery for treatment of obstructive sleep apnea. J Oral Maxillofacial Surgery 2008;66:968-72.

94. Veasey S, Guilleminault C, Strohl K, et al. Medical therapy for obstructive sleep apnea: a review by the Medical Therapy for Obstructive Sleep Apnea Task Force of the Standards of Practice Committee of the American Academy of Sleep Medicine. Sleep 2006;29:1036-44. 